\title{
WAVE PROPAGATION IN AN ELASTIC SOLID WITH A LINE OF DISCONTINUITY OR FINITE CRACK*
}

\author{
BY \\ G. C. SIH \\ (Lehigh University) \\ AND \\ J. F. LOEBER \\ (Knolls Atomic Power Laboratory, Schenectady, New York)
}

\begin{abstract}
With the aid of integral transforms, a method is presented for solving the problem of scattering of plane harmonic compression and shear waves by a line of discontinuity or crack of finite width embedded in an elastic medium of infinite extent. When the incoming waves are applied in an arbitrary direction, the scattered-wave field may be determined by separating the crack-surface boundary conditions into functions even and odd with respect to the variable along the line crack. The problem is reduced to the evaluation of a system of coupled Fredholm integral equations with special emphasis placed on finding the near-field solution which consists of a knowledge of the detailed structure of the displacements and stresses in a small region around the crack vertex. Dynamic stress-intensity factors, the critical values of which govern the condition of crack propagation, are defined and found to be dependent on the incident wave length and Poisson's ratio of the medium. At certain wave lengths, they are larger than those encountered under static loading. Such information is of particular importance in perdicting the fracture strength of structures subjected to oscillating loads.

Introduction. Although the scattering of waves by obstacles of different shapes has been the subject of many past investigations in various branches of physics [1]-[3], to the authors' knowledge none of these investigations analyzed, in detail, the singular behavior of the stresses near a scatterer in the form of a line of discontinuity or finite crack. The main reason for this omission is the lack of an effective mathematical method for obtaining the near-field solution, which is of considerable theoretical interest and has innumerable applications in the field of fracture mechanics as well as in electromagnetic and acoustic theory.

A popular approach to the diffraction of waves from obstacles has been that of separation of variables, where the formal solution of the wave equation is given by an infinite series of orthogonal functions. Such an approach, however, is effective only for obstacle shapes adapted to those coordinate systems in which the wave equation is separable. For this reason, the dynamic stress concentrations around circular and parabolic obstacles have received considerable attention in the past. A comprehensive survey of the literature in a field as wide and diversified as the propagation of elastic waves is clearly beyond the scope of this paper. In recent years, the Mow-Pao-Thau school [4]-[6] has
\end{abstract}

\footnotetext{
* Received January 8, 1968; revised version received March 29, 1968. The research described in this paper was sponsored by the U. S. Navy under Contract Nonr-610(06) with the Office of Naval Research, Washington, D. C.
} 
published a number of papers on this subject. References to other work can be found in [4]-[6].

It is well known that problems involving diffraction of plane harmonic, horizontally polarized shear waves ( $\mathrm{SH}$-waves) by a semi-infinite crack can be formulated in terms of integral equations, and solved by the Wiener-Hopf technique [7]. As pointed out by Sih [8], however, since the static limit of the semi-infinite crack solution is zero, it is not possible to estimate the precise magnification of the stresses due to dynamic effects. To overcome this shortcoming, Loeber and Sih [9] proposed to add another characteristic dimension into the problem, namely the crack width, and managed to obtain the exact behavior of the crack-front displacement and stress fields for the case of SH-waves diffracted by a finite or internal crack. Ang and Knopoff [10] have attempted to solve the internal crack problem earlier but their method yields results which are restricted to low frequencies and to distances far away from the crack. In elastodynamics, the farfield crack solution is not useful in the sense that it offers no information to the development of the theories of crack propagation. Generally speaking, the far-field solution can always be determined by the standard method of Wiener-Hopf [7] in a straightforward manner. On the other hand, considerable difficulty is encountered when the WienerHopf method is applied to find the near-field solution. One of the difficulties arises from the factorization of certain functions into functions analytic in the upper and lower half planes. The problem of the diffraction of electromagnetic waves ${ }^{1}$ incident upon a slit has also been treated by Schmeltzer and Lewin [11] using the function-theoretic approach. Their results are left in terms of several complicated integrals the evaluation of which becomes a problem in itself, particularly in seeking the analytical form of the solution in the vicinity of the slit.

Having discussed the previous work related to crack problems of SH-waves, it is natural to follow the discussion with a few remarks concerning the diffractions of plane harmonic compression waves (P-waves) and vertically polarized shear waves (SV-waves) by a line crack. Although both Miles [12] and Papadopoulos [13] have investigated crack problems of this type, their work discusses only the qualitative character of the displacement potentials without any explicit information given as to the nature of the local stress distribution. The mathematical description of these problems is somewhat complex because the scattered waves, caused by the line crack, are composed of both compression and shear waves even though the input wave may be of one type, either the P- or SVwaves. For this and other reasons, the near-field solution of waves scattered by a crack with finite width is yet to be found.

The purpose of this paper, aside from obtaining the stress solution close to the crack point, is to offer a method of solution for solving diffraction problems involving $\mathrm{P}$ - and SV-waves incident upon a line of discontinuity. The method can handle different types of boundary conditions ${ }^{2}$ on the line of discontinuity. For illustration, only the case of a traction-free crack will be considered. An important conclusion is that within certain ranges of wave lengths the dynamic stress distribution around the crack is quite sensitive to changes in the wave number. This is displayed graphically for different values of the

${ }^{1}$ The scattering of plane-polarized electromagnetic waves by a screen in a flitid medium is mathematically analogous to the SH-wave crack problem in elastodynamics.

${ }^{2} \mathrm{By}$ following the steps outlined in this paper, it is clear that the problems of a rigid and rigidsmooth strip can be solved in the same way. 
Poisson's ratio. The knowledge gained in this investigation is believed to add further impetus to the understanding of the propagation of cracks under fluctuating loads.

Field equations and input waves. Consider the propagation of elastic waves, produced by the action of oscillating compressional and shear forces, which vary harmonically in time and are applied in the $x y$-plane containing a through crack. In the plane, there arise both compressional and shear waves, and the resulting displacements can be expressed in terms of two scalar functions $\phi$ and $\psi$ each of which depend upon $x, y$, and $t$. The rectangular components of the displacement vector are

$$
u_{x}=\partial \phi / \partial x+\partial \psi / \partial y, u_{y}=\partial \phi / \partial y-\partial \psi / \partial x,
$$

Substituting Eq. (1) into the equations of motion under the conditions of plane strain, the following wave equations on $\phi$ and $\psi$ are obtained:

$$
c_{1}^{2}\left(\frac{\partial^{2} \phi}{\partial x^{2}}+\frac{\partial^{2} \phi}{\partial y^{2}}\right)=\frac{\partial^{2} \phi}{\partial t^{2}}, \quad c_{2}^{2}\left(\frac{\partial^{2} \psi}{\partial x^{2}}+\frac{\partial^{2} \psi}{\partial y^{2}}\right)=\frac{\partial^{2} \psi}{\partial t^{2}} .
$$

In Eq. (2), $c_{1}$ and $c_{2}$ stand, respectively, for the velocities of compression (irrotational) and shear (equivoluminal) waves in an infinitely extended elastic medium; they are given by

$$
c_{1}=[(\lambda+2 \mu) / \rho]^{1 / 2}, \quad c_{2}=(\mu / \rho)^{1 / 2}
$$

with $\rho$ being the mass density. As usual, in the case of generalized plane stress the Lamé constant $\lambda$ in Eq. (3) is to be replaced by $2 \lambda \mu /(\lambda+2 \mu)$, while the shear modulus of elasticity $\mu$ remains unchanged. From the stress and displacement relations, it is found that

$$
\begin{aligned}
& \sigma_{x x}=\lambda \nabla^{2} \phi+2 \mu\left(\frac{\partial^{2} \phi}{\partial x^{2}}+\frac{\partial^{2} \psi}{\partial x \partial y}\right), \\
& \sigma_{y \nu}=\lambda \nabla^{2} \phi+2 \mu\left(\frac{\partial^{2} \phi}{\partial y^{2}}-\frac{\partial^{2} \psi}{\partial x \partial y}\right), \\
& \sigma_{x y}=\mu\left(2 \frac{\partial^{2} \phi}{\partial x \partial y}-\frac{\partial^{2} \psi}{\partial x^{2}}+\frac{\partial^{2} \psi}{\partial y^{2}}\right) .
\end{aligned}
$$

With reference to the rectangular coordinate system $x, y$ of Fig. 1, let a line crack be

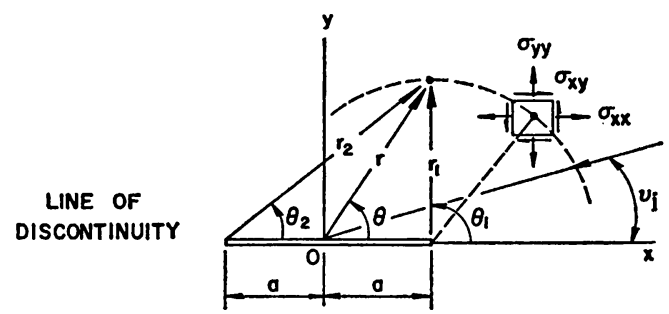

Fig. 1. Polar coordinates on line of discontinuity.

placed along the $x$-axis from $-a$ to $+a$, and an incident plane harmonic compressional wave ( $\mathrm{P}$-wave) be directed at an angle $v_{1}$ with the $x$-axis so that 


$$
\begin{aligned}
& \left.\phi^{(i)}=\phi_{0} \exp \left\{-i \mid \alpha_{1}\left(x \cos v_{1}+y \sin v_{1}\right)+\omega t\right]\right\}, \\
& \psi^{(i)}=0
\end{aligned}
$$

in which $\phi_{0}$ is a measure of the wave amplitude, $\omega$ is the circular frequency, and $\alpha_{1}=$ $\omega / c_{1}$ is the wave number for compression waves.

In preparation for subsequent work, the stresses of the incident P-waves will be computed by inserting Eq. (5) into (4); the results are

$$
\begin{aligned}
& \sigma_{x x}^{(i)}=-\mu \alpha_{2}^{2} \phi^{(i)}\left(1-2 \kappa^{2} \sin ^{2} v_{1}\right), \\
& \sigma_{\nu y}^{(i)}=-\mu \alpha_{2}^{2} \phi^{(i)}\left(1-2 \kappa^{2} \cos ^{2} v_{1}\right), \\
& \sigma_{x y}^{(i)}=-\mu \alpha_{2}^{2} \phi^{(i)} \kappa^{2} \sin 2 v_{1},
\end{aligned}
$$

where the elastic constant $\kappa^{2}=\left(c_{2} / c_{1}\right)^{2}$ takes the value $(1-2 \nu) / 2(1-\nu)$ for plane strain and $(1-\nu) / 2$ for generalized plane stress. The maximum value of the normal stress acting on the incident plane compression wave front is $-\mu \alpha_{2}^{2} \phi_{0}$ which is assumed to remain finite as $\alpha_{1} \rightarrow 0$.

Similarly, if an incident plane harmonic shear wave (SV-wave) impinges on the crack at an angle $v_{2}$ with the $x$-axis, then

$$
\begin{aligned}
& \phi^{(i)}=0, \\
& \psi^{(i)}=\psi_{0} \exp \left\{-i\left[\alpha_{2}\left(x \cos v_{2}+y \sin v_{2}\right)+\omega t\right]\right\} .
\end{aligned}
$$

and the corresponding stress field is

$$
\begin{aligned}
& \sigma_{x x}^{(i)}=-\mu \alpha_{2}^{2} \psi^{(i)} \sin 2 v_{2}, \\
& \sigma_{y y}^{(i)}=-\sigma_{x x}^{(i)}, \\
& \sigma_{x y}^{(i)}=\mu \alpha_{2}^{2} \psi^{(i)} \cos 2 v_{2},
\end{aligned}
$$

in which $\mu \alpha_{2}^{2} \psi_{0}$ is the maximum shear stress acting on the shear wave front and is nonzero as $\alpha_{2} \rightarrow 0$. The quantity $\psi_{0}$ is a constant, and $\alpha_{2}=\omega / c_{2}$ is the shear-wave number.

In view of the harmonic time-variation of the input waves given by Eqs. (5) and (7), the displacements, stresses, and potentials $\phi$ and $\psi$ will all contain the time factor $\exp (-i \omega t)$ which will henceforth be dropped.

Since both Eqs. (5) and (7) are already solutions of the wave equations, the main burden of the analysis is to determine the potentials $\phi^{(r)}$ and $\psi^{(r)}$ of the scattered-wave field governed by the Helmholtz equations

$$
\begin{gathered}
\frac{\partial^{2} \phi^{(r)}}{\partial x^{2}}+\frac{\partial^{2} \phi^{(r)}}{\partial y^{2}}+\alpha_{1}^{2} \phi^{(r)}=0, \\
\frac{\partial^{2} \psi^{(r)}}{\partial x^{2}}+\frac{\partial^{2} \psi^{(r)}}{\partial y^{2}}+\alpha_{2}^{2} \psi^{(r)}=0
\end{gathered}
$$

for problems involving steady-state motion. The total wave field may then be taken as the linear sum of the displacement potentials of the incident and diffracted waves as

$$
\phi=\phi^{(i)}+\phi^{(r)}, \quad \psi=\psi^{(i)}+\psi^{(r)},
$$


where

$$
\phi^{(r)}, \psi^{(r)} \rightarrow 0 \quad \text { as } \quad\left(x^{2}+y^{2}\right)^{1 / 2} \rightarrow \infty .
$$

The same general relationships apply to the total displacement and stress fields.

For a traction-free crack surface, the total normal and shear stresses at $y=0$ and $|x|<a$ must vanish, i.e.,

$$
\begin{aligned}
& \sigma_{y y}^{(i)}(x, 0)+\sigma_{y y}^{(r)}(x, 0)=0, \quad|x|<a, \\
& \sigma_{x y}^{(i)}(x, 0)+\sigma_{x y}^{(r)}(x, 0)=0, \quad|x|<a
\end{aligned}
$$

from which the boundary conditions of the scattered-wave problem may be established. For convenience, the problem will be split into two parts, namely, that of finding the solution of Eqs. (9) for the following conditions:

Case A.

$$
\begin{array}{ll}
u_{y}^{(r)}(x, 0)=\sigma_{x y}^{(r)}(x, 0)=0, & |x|>a, \\
\sigma_{y y}^{(r)}(x, 0)=-\sigma_{y y}^{(i)}(x, 0) ; & \sigma_{x y}^{(r)}(x, 0)=0, \quad|x|<a .
\end{array}
$$

Case B.

$$
\begin{aligned}
& u_{x}^{(r)}(x, 0)=\sigma_{y y}^{(r)}(x, 0)=0, \quad|x|>a, \\
& \sigma_{y y}^{(r)}(x, 0)=0 ; \quad \sigma_{x y}^{(r)}(x, 0)=-\sigma_{x y}^{(i)}(x, 0), \quad|x|<a .
\end{aligned}
$$

Clearly, the stress solutions for Cases A and B are even and odd in $y$, respectively; consequently, the former case will be called the symmetric problem and the latter the skew-symmetric problem. The desired solution of the original problem can be obtained by superposition of the solutions for the two Cases. Because of the symmetry conditions in Eqs. (10) and (11), it is possible, in each case, to lay down certain conditions on the plane $y=0$, and consider the problem for the half-plane, $y \geq 0$, only.

Case A-symmetric problem. The resulting stresses of the scattered waves are said to be symmetric with respect to the crack plane $y=0$, if the crack is opened ${ }^{3}$ by oppositely acting normal tractions. These normal tractions are taken to be the negative of those shown in equations (6) and (8), i.e., ${ }^{4}$

$$
\sigma_{\nu \nu}^{(r)}(x, 0)=-\sigma_{y y}^{(i)}(x, 0)=(-1)^{i} 2 \mu P_{i} \exp \left(-i \alpha_{i} x \cos v_{i}\right), \quad|x|<a,
$$

where $j=1,2$ such that

$$
P_{1}=-\frac{1}{2} \alpha_{2}^{2} \phi_{0}\left(1-2 \kappa^{2} \cos v_{1}\right)
$$

corresponds to the incident $\mathrm{P}$-wive and

$$
P_{2}=\frac{1}{2} \alpha_{2}^{2} \psi_{1} \sin 2 v_{2}
$$

to the incident SV-wave. To make use of the Fourier sine and cosine transforms, the problem will be further divided into parts that are even and odd in $x$ by separating the real and imaginary parts of Eq. (12).

${ }^{3}$ When the tractions change in sign, it is obvious that on the compression cycle the two sides of the crack will come in contact with each other. Such a violation of the free-surface condition, however, is not serious, and can be easily corrected. A detailed account of this point is reserved for later discussion.

${ }^{4}$ The factor $(-1)^{i}(j=1,2)$ is defined for values of $0 \leq v_{2} \leq \pi / 4$. Appropriate changes should be made for other values of $v_{2}$, say $\pi / 4<v_{2} \leq \pi / 2$. 
1. Normal Tractions Even in $x$. Taking into account only the real part of $\sigma_{v y}^{(r)}(x, 0)$, which is even in $x$, the potentials $\phi^{(r)}$ and $\psi^{(r)}$ may be represented by the cosine and sine integrals

$$
\begin{array}{ll}
\phi^{(r)}(x, y)=\frac{2}{\pi} \int_{0}^{\infty} A_{1}(s) \exp \left(-\beta_{1} y\right) \cos (s x) d s, & y \geq 0 . \\
\psi^{(r)}(x, y)=\frac{2}{\pi} \int_{0}^{\infty} A_{2}(s) \exp \left(-\beta_{2} y\right) \sin (s x) d s, & y \geq 0 .
\end{array}
$$

The branch cuts of the functions

$$
\beta_{i}=\left(s^{2}-\alpha_{j}^{2}\right)^{1 / 2}=-i\left(\alpha_{i}^{2}-s^{2}\right)^{1 / 2}, \quad j=1,2
$$

have already been discussed in [7] and thus no additional comments are needed. Applying the boundary conditions in Eqs. (10) to the appropriate expressions of displacement and stress obtained from putting Eqs. (13) into Eqs. (1) and (2), the results may be written in the forms

$$
\begin{aligned}
& \frac{2}{\pi} \int_{0}^{\infty}\left[-\beta_{1} A_{1}(s)-s A_{2}(s)\right] \cos (s x) d s=0, \quad|x|>a \\
& \frac{2}{\pi} \int_{0}^{\infty}\left[\frac{1}{s}\left(s^{2}-\frac{1}{2} \alpha_{2}^{2}\right)^{2} A_{1}(s)+\beta_{2} A_{2}(s)\right] \sin (s x) d s \\
& =\frac{(-1)^{i} P_{i} \sin \left(\alpha_{i} x \cos v_{j}\right)}{\alpha_{i} \cos v_{i}}, \quad|x|<a
\end{aligned}
$$

and the condition of $\sigma_{x y}^{(r)}(x, 0)=0$ for all values of $x$ is satisfied by setting

$$
s \beta_{1} A_{1}(s)+\left(s^{2}-\frac{1}{2} \alpha_{2}^{2}\right) A_{2}(s)=0 .
$$

Now, define a function $A(s)$ through

$$
A(s)=-\beta_{1} A_{1}(s)-s A_{2}(s)
$$

so that

$$
\left[\begin{array}{c}
A_{1}(s) \\
A_{2}(s)
\end{array}\right]=\frac{2 A(s)}{\alpha_{2}^{2} \beta_{1}}\left[\begin{array}{c}
\left(s^{2}-\frac{1}{2} \alpha_{2}^{2}\right) \\
-s \beta_{1}
\end{array}\right]
$$

Under these considerations, it can be confirmed from Eqs. (14) that $A(s)$ is governed by the pair of dual integral equations

$$
\begin{aligned}
\frac{2}{\pi} \int_{0}^{\infty} A(s) \cos (s x) d s & =0, \quad|x|>a \\
\frac{2}{\pi} \int_{0}^{\infty} f(s) A(s) \sin (s x) d s & =\frac{(-1)^{i} P_{i} \sin \left(\alpha_{i} x \cos v_{i}\right)}{\alpha_{i} \cos v_{i}}, \quad|x|<a
\end{aligned}
$$

in which

$$
f(s)=\frac{2}{\alpha_{2}^{2} \beta_{1} s}\left[\left(s_{2}-\frac{1}{2} \alpha_{2}^{2}\right)^{2}-s^{2} \beta_{1} \beta_{2}\right] .
$$

The objective is to reduce the problem to an integral equation of a standard type. 
Introduce the function

$$
a(x)=\frac{2}{\pi} \int_{0}^{\infty} A(s) \cos (s x) d s
$$

and by the Fourier inversion theorem, it is obvious that

$$
A(s)=\int_{0}^{\infty} a(x) \cos (s x) d x .
$$

According to the first of Eqs. (15), $a(x)$ vanishes for $|x|>a$ and thus

$$
A(s)=\int_{0}^{a} a(x) \cos (s x) d x
$$

Elimination of $A(s)$ from the second of Eqs. (15) then gives

$$
\frac{2}{\pi} \int_{0}^{\infty} f(s) \sin (s x) d s \int_{0}^{a} a(\tau) \cos (s \tau) d \tau=\frac{(-1)^{i} P_{i} \sin \left(\alpha_{j} x \cos v_{i}\right)}{\alpha_{i} \cos v_{i}}, \quad|x|<a
$$

which involves $a(\tau)$ as the only unknown function.

From the physics of the crack problem [14], the displacements at the end points $x= \pm a$ of the crack must be bounded and are of the order $(x \mp a)^{1 / 2}$ as $|x| \rightarrow a$, i.e.,

$$
a(x)=\lambda(a)\left(a^{2}-x^{2}\right)^{1 / 2}+O(1), \text { as }|x| \rightarrow a .
$$

Guided by Eq. (17), it is assumed that $a(x)$ admits the representation

$$
a(x)=\int_{x}^{a} \lambda(\tau) \frac{\tau d \tau}{\left(\tau^{2}-x^{2}\right)^{1 / 2}}, \quad 0 \leq x \leq a
$$

where $\lambda(\tau)$ is required to be continuous on the interval [0,a]. Equation (18) may be integrated by parts to give

$$
a(x)=\lambda(a)\left(a^{2}-x^{2}\right)^{1 / 2}-\int_{x}^{a} \lambda^{\prime}(\tau)\left(\tau^{2}-x^{2}\right)^{1 / 2} d \tau, \quad 0 \leq x \leq a
$$

which agrees with the preassigned form of $a(x)$ in Eq. (17). In what follows, the function $\lambda(\tau)$ is permitted to depend on the physical parameters in the problem.

Substituting Eq. (18) into (16), and applying the Dirichlet formula for interchanging the order of integration [15] render

$$
\int_{0}^{\infty} f(s) \sin (s x) d s \int_{0}^{a} \tau \lambda(\tau) J_{0}(s \tau) d \tau=\frac{(-1)^{i} P_{i} \sin \left(\alpha_{i} x \cos v_{i}\right)}{\alpha_{i} \cos v_{i}},|x|<a
$$

where use was made of the identity [16]

$$
\int_{0}^{\tau} \frac{\cos (s x) d x}{\left(\tau^{2}-x^{2}\right)^{1 / 2}}=\frac{\pi}{2} J_{0}(s \tau)
$$

Here, $J_{0}$ is the zero-order Bessel function of the first kind. For the purpose of reducing Eq. (19) to a standard Fredholm equation, let

$$
g(s)=f(s)+\left(1-\kappa^{2}\right) \rightarrow O\left(s^{-2}\right), \text { as } s \rightarrow \infty
$$

and recall the discontinuous integral

$$
\int_{0}^{\infty} J_{0}(s \tau) \sin (s x) d s= \begin{cases}0, & 0 \leq x<\tau \\ 1 /\left(x^{2}-\tau^{2}\right)^{1 / 2}, & 0<\tau<x\end{cases}
$$


After a permissible reversal of the order of integrations with respect to $s$ and $\tau$, Eq. (19) takes the form of Abel's equation [15]

$$
\int_{0}^{x} \lambda(\tau) \frac{\tau d \tau}{\left(x^{2}-\tau^{2}\right)^{1 / 2}}=h(x)
$$

provided

$h(x)=\left(1-\kappa^{2}\right)^{-1}\left[\frac{(-1)^{i-1} P_{j} \sin \left(\alpha_{i} x \cos v_{i}\right)}{\alpha_{i} \cos v_{i}}+\int_{0}^{a} \tau \lambda(\tau) d \tau \int_{0}^{\infty} g(s) J_{0}(s \tau) \sin (s x) d s\right]$.

The Abel equation may be inverted to free $\lambda(\tau)$ from the integration, i.e.,

$$
\lambda(\tau)=\frac{2}{\pi} \int_{0}^{\tau} \frac{h^{\prime}(x) d x}{\left(\tau^{2}-x^{2}\right)^{1 / 2}}, \quad 0 \leq \tau \leq a .
$$

The result of inserting the derivative of $h(x)$ into Eq. (21) is

$$
\begin{aligned}
\lambda(\tau)=\left(1-\kappa^{2}\right)^{-1}\left[(-1)^{j-1} P_{i} J_{0}\left(\alpha_{i} \tau \cos v_{i}\right)\right. & \\
& \left.+\int_{0}^{a} \zeta \lambda(\zeta) d \zeta \int_{0}^{\infty} s g(s) J_{0}(s \tau) J_{0}(s \zeta) d s\right], \quad 0 \leq \tau \leq a
\end{aligned}
$$

whose kernel may be symmetrized by the introduction of the dimensionless quantities

$$
\xi=\frac{\tau}{a}, \eta=\frac{\zeta}{a}, \Lambda(\xi)=\frac{(-1)^{i}\left(1-\kappa^{2}\right) \xi^{1 / 2} \lambda(a \xi)}{P_{i}}, \quad 0<\xi \leq 1 .
$$

This leads to a regular integral equation of Fredholm's second kind

$$
\Lambda(\xi)-\int_{0}^{1} \Lambda(\eta) F(\xi, \eta) d \eta=(-1)^{i} \xi^{1 / 2} J_{0}\left(\alpha_{j} a \xi \cos v_{i}\right), \quad 0<\xi \leq 1,
$$

where

$F(\xi, \eta)=\left(1-\kappa^{2}\right)^{-1}(\xi \eta)^{1 / 2} \int_{0}^{\infty} s g\left(\frac{s}{a}\right) J_{0}(s \xi) J_{0}(s \eta) d s, \quad 0<\xi \leq 1 ; \quad 0<\eta \leq 1$.

In view of $\beta_{i}=\left(s^{2}-\alpha_{j}^{2}\right)^{1 / 2}(j=1,2)$ (connected with the out-going waves) being complex, both $\Lambda(\xi)$ and $F(\xi, \eta)$ in equation (22) are also complex functions. Separating these functions into their real and imaginary parts in accordance with

$$
\Lambda(\xi)=\Lambda_{1}(\xi)+i \Lambda_{2}(\xi), \quad F(\xi, \eta)=F_{1}(\xi, \eta)+i F_{2}(\xi, \eta)
$$

the following system of coupled integral equations are obtained:

$$
\begin{gathered}
\Lambda_{1}(\xi)-\int_{0}^{1}\left[\Lambda_{1}(\eta) F_{1}(\xi, \eta)-\Lambda_{2}(\eta) F_{2}(\xi, \eta)\right] d \eta=(-1)^{j} \xi^{1 / 2} J_{0}\left(\alpha_{j} a \xi \cos v_{i}\right), \\
\Lambda_{2}(\xi)-\int_{0}^{1}\left[\Lambda_{1}(\eta) F_{2}(\xi, \eta)+\Lambda_{2}(\eta) F_{1}(\xi, \eta)\right] d \eta=0, \quad 0<\xi \leq 1 .
\end{gathered}
$$

The kernels $F_{i}(\xi, \eta)(j=1,2)$ are given by

$$
\begin{aligned}
& F_{1}(\xi, \eta)=\frac{2}{\alpha_{2}^{2}\left(1-\kappa^{2}\right)}(\xi \eta)^{1 / 2} \int_{0}^{\infty} s\left[f_{1}\left(\frac{s}{a}\right)-g_{2}\left(\frac{s}{a}\right)+\frac{1}{2} \alpha_{2}^{2}\left(1-\kappa^{2}\right)\right] \\
& F_{2}(\xi, \eta)=\frac{2}{\alpha_{2}^{2}\left(1-\kappa^{2}\right)}(\xi \eta)^{1 / 2} \int_{0}^{\infty} s\left[m_{1}\left(\frac{s}{a}\right)+n_{2}\left(\frac{s}{a}\right)\right] J_{0}(s \eta) d s,
\end{aligned}
$$


where $0<\xi \leq 1,0<\eta \leq 1$, and

$$
\begin{aligned}
& f_{k}(s)=s^{-1}\left(s^{2}-\frac{1}{2} \alpha_{2}^{2}\right)^{2} \begin{cases}0, & s<\alpha_{k} \\
1 /\left(s^{2}-\alpha_{k}^{2}\right), & s>\alpha_{k}\end{cases} \\
& g_{l}(s)=s\left\{\begin{array}{lc}
0, & s<\alpha_{l} \\
\left(s^{2}-\alpha_{l}^{2}\right)^{1 / 2}, & s>\alpha_{l}
\end{array}\right. \\
& m_{k}(s)=s^{-1}\left(s^{2}-\frac{1}{2} \alpha_{2}^{2}\right)^{2} \begin{cases}1 /\left(\alpha_{k}^{2}-s^{2}\right)^{1 / 2} & s<\alpha_{k} \\
0, & s>\alpha_{k}\end{cases} \\
& n_{l}(s)=s \begin{cases}\left(\alpha_{l}^{2}-s^{2}\right)^{1 / 2}, & s<\alpha_{l} \\
0, & s>\alpha_{l} .\end{cases}
\end{aligned}
$$

The above format is employed with the knowledge that the solutions for the scattering problem of $\mathrm{P}$ - and SV-waves are obtained, respectively, by setting $k=1, l=2$ and $k=2, l=1$.

To improve the rate of convergence of the numerical calculations, it is desirable to evaluate the singular parts of the integrals in Eqs. (25) in closed form by letting

$$
p(s)=1+2 \frac{f_{1}(s)-g_{2}(s)}{\alpha_{2}^{2}\left(1-\kappa^{2}\right)}-\frac{M}{s^{2}+m^{2}}
$$

in which $m$ and $M$ stand for

$$
m^{2}=\frac{1}{2} \alpha_{2}^{2} \cdot\left(\frac{1+2 \kappa^{2}-6 \kappa^{4}+5 \kappa^{6}}{3-4 \kappa^{2}+3 \kappa^{4}}\right), \quad M=\frac{1}{4} \alpha_{2}^{2} \cdot\left(\frac{3-4 \kappa^{2}+3 \kappa^{4}}{1-\kappa^{2}}\right) .
$$

With the aid of the identity [16]

$$
\int_{0}^{\infty} \frac{s}{s^{2}+m^{2}} J_{0}(\xi s) J_{0}(\eta s) d s=I_{0}(m \xi) K_{0}(m \eta), \quad 0<\xi \leq \eta,
$$

where $I_{0}$ and $K_{0}$ are the modified zero-order Bessel functions of the first and second kind, respectively, $F_{1}(\xi, \eta)$ becomes

$$
\begin{aligned}
& F_{1}(\xi, \eta)=(\xi \eta)^{1 / 2}\left[a^{2} M I_{0}(a m \xi) K_{0}(a m \eta)\right. \\
& \left.\quad+\int_{0}^{\infty} s p\left(\frac{s}{a}\right) J_{0}(s \xi) J_{0}(s \eta) d s\right], \quad 0<\xi \leq \eta \leq 1
\end{aligned}
$$

and its continuity on $[0 \leq \xi \leq 1,0 \leq \eta \leq 1]$ is assured by the behavior of $I_{0}$ and $K_{0}$ in a neighborhood of the origin. The advantage of this alternative representation of $F_{1}(\xi, \eta)$, as compared with the first of Eqs. (25), is apparent from

$$
p(s) \rightarrow O\left(s^{-6}\right), \text { as } s \rightarrow \infty,
$$

The system of integral equations for $\Lambda_{i}(\xi)(j=1,2)$ may be solved numerically on an electronic computer. With the knowledge of $\Lambda(\xi)$, the only unknown $A(s)$ in the scattering problem with normal tractions even in $x$ can be evaluated:

$$
A(s)=(-1)^{j} \frac{\pi P_{i} a}{2\left(1-\kappa^{2}\right) s}\left\{\Lambda(1) J_{1}(s a)-\int_{0}^{1} \xi J_{1}(a \xi s)\left[\frac{\Lambda(\xi)}{\xi^{1 / 2}}\right]^{\prime} d \xi\right\} .
$$


The displacements and stresses everywhere in the elastic medium with a crack can then be calculated without difficulty. As mentioned in the Introduction, this method of solution is particularly suited for obtaining the explicit form of the crack-tip stress field, which together with the numerical results for $\Lambda(\xi)$ will be presented subsequently.

2. Normal Tractions Odd in $x$. Suppose that the normal tractions on the crack are given by the imaginary part of $\sigma_{y y}^{(r)}(x, 0)$ in Eq. (12). Then the correct evenness and oddness properties in $x$ for the potentials of the scattered waves may be represented by

$$
\begin{array}{ll}
\phi^{(r)}(x, y)=\frac{2}{\pi} \int_{0}^{\infty} B_{1}(s) \exp \left(-\beta_{1} y\right) \sin (s x) d s, & y \geq 0, \\
\psi^{(r)}(x, y)=\frac{2}{\pi} \int_{0}^{\infty} B_{2}(s) \exp \left(-\beta_{2} y\right) \cos (s x) d s, & y \geq 0 .
\end{array}
$$

As before, the boundary conditions in Eqs. (10) lead to a pair of dual integral equations

$$
\begin{gathered}
\frac{2}{\pi} \int_{0}^{\infty} B(s) \cos (s x) d s=0, \quad|x|>a, \\
\frac{2}{\pi} \int_{0}^{\infty} f(s) B(s) \sin (s x) d s=i(-1)^{i-1} P_{i} \sin \left(\alpha_{i} x \cos v_{i}\right), \quad|x|<a
\end{gathered}
$$

in which $B(s)$ is connected with $B_{i}(s)(j=1,2)$ by

$$
\left[\begin{array}{l}
B_{1}(s) \\
B_{2}(s)
\end{array}\right]=\frac{2 B(s)}{\alpha_{2}^{2} \beta_{1} s}\left[\begin{array}{c}
\left(s^{2}-\frac{1}{2} \alpha_{2}^{2}\right) \\
s \beta_{1}
\end{array}\right] .
$$

Making use of the first of Eqs. (29) and the Fourier inversion theorem, $B(s)$ may be linked to a function $b(x)$, i.e.,

$$
B(s)=\int_{0}^{a} b(x) \cos (s x) d s .
$$

The singularities to be expected in the function $b(x)$ will be pre-assigned. Knowing the nature of the stress singularities at the endpoints of the crack [14], it will be assumed that

$$
b(x)=\frac{a \delta^{*}}{\left(a^{2}-x^{2}\right)^{1 / 2}}+\int_{x}^{a} \frac{\gamma(\tau) d \tau}{\left(\tau^{2}-x^{2}\right)^{1 / 2}}, \quad 0 \leq x<a .
$$

Here $\delta^{*}$ is a constant and $\gamma(\tau)$ a function continuous on the interval $[0, a]$. This implies

$$
b(s)=\frac{a \delta^{*}}{\left(a^{2}-x^{2}\right)^{1 / 2}}+O(1), \quad \text { as } x \rightarrow a ; \quad 0 \leq x<a
$$

which preserves the $(x \mp a)^{-1 / 2}$-type of stress singularities at the crack tips, $x= \pm a$, respectively. The function $B(s)$ may now be expressed in terms of the newly introduced function $\gamma(\tau)$ as

$$
B(x)=\frac{\pi}{2}\left[a \delta^{*} J_{0}(s a)+\int_{0}^{a} J_{0}(s \tau) \gamma(\tau) d \tau\right] .
$$

The constant $\delta^{*}$ can be evaluated from the condition that the displacements at the crack tips are bounded, i.e.,

$$
\lim _{x \rightarrow a} \int_{0}^{x} b(x) d x=0
$$


which gives

$$
\delta^{*}=-\frac{1}{a} \int_{0}^{a} \gamma(\tau) d \tau
$$

Inserting Eq. (31) into the second of Eqs. (29), and rearranging the subsequent result yield an integral equation of the Abel type:

$$
\begin{aligned}
\int_{0}^{x} \frac{\gamma(\tau)}{\left(x^{2}-\frac{d \tau}{\left.\tau^{2}\right)^{1 / 2}}\right.}=\left(1-\kappa^{-2}\right)\left\{i(-1)^{i} P_{i} \sin \left(\alpha_{j} x \cos v_{i}\right)\right. \\
\left.+\int_{0}^{\infty} g(s)\left[a \delta^{*} J_{0}(s a)+\int_{0}^{a} J_{0}(s \tau) \gamma(\tau) d \tau\right] \sin (s x) d s\right\}, \quad 0 \leq x<a .
\end{aligned}
$$

The inverted form of the Abel equation is

$$
\begin{aligned}
\gamma(\tau)=(1 & \left.-\kappa^{2}\right)^{-1} \tau\left\{i(-1)^{i} P_{i} \alpha_{j} \cos v_{j} J_{0}\left(\alpha_{i} \tau \cos v_{i}\right)\right. \\
& \left.+\int_{0}^{\infty} s g(s)\left[a \delta^{*} J_{0}(s a)+\int_{0}^{a} J_{0}(s \zeta) \gamma(\zeta) d \zeta\right] J_{0}(s \tau) d s\right\}, \quad 0<\tau \leq a .
\end{aligned}
$$

With the aim of symmetrizing the kernel in Eq. (33), introduce the change of variables $\xi=\tau / a, \eta=\zeta / a$, and set

$$
\left[\begin{array}{c}
\delta^{*} \\
\gamma(\tau)
\end{array}\right]=\frac{(-1)^{i} P_{j} \cos v_{j}}{1-\kappa^{2}}\left[\begin{array}{c}
\delta \\
\xi^{1 / 2} \Gamma(\xi)
\end{array}\right], \quad 0<\xi \leq 1 .
$$

Thus, Eq. (33) assumes the form of a Fredholm integral equation

$$
\Gamma(\xi)-\int_{0}^{1} \Gamma(\eta) F(\xi, \eta) d \eta=i(-1)^{i-1} \alpha_{i} a \xi^{1 / 2} J_{0}\left(\alpha_{i} a \xi \cos v_{i}\right)+\delta F(\xi, 1), \quad 0<\xi \leq 1,
$$

where the kernel $F(\xi, \eta)$ is the same as that shown in equation (23), and

$$
\delta=-\int_{0}^{1} \xi^{1 / 2} \Gamma(\xi) d \xi
$$

Since $F(\xi, \eta)$ and

$$
\Gamma(\xi)=\Gamma_{1}(\xi)+i \Gamma_{2}(\xi)
$$

are complex, Eq. (34) may be further decomposed into real and imaginary parts:

$$
\begin{aligned}
& \Gamma_{1}(\xi)-\int_{0}^{1}\left[\Gamma_{1}(\eta) F_{1}(\xi, \eta)-\Gamma_{2}(\eta) F_{2}(\xi, \eta)\right] d \eta \\
& =-\int_{0}^{1}\left[\Gamma_{1}(\eta) F_{1}(\xi, 1)-\Gamma_{2}(\eta) F_{2}(\xi, 1)\right] \eta^{1 / 2} d \eta, \quad 0<\xi \leq 1 \\
& \Gamma_{2}(\xi)-\int_{0}^{1}\left[\Gamma_{1}(\eta) F_{2}(\xi, \eta)+\Gamma_{2}(\eta) F_{1}(\xi, \eta)\right] d \eta \\
& =(-1)^{i-1} \alpha_{j} a \xi^{1 / 2} J_{0}\left(\alpha_{j} a \xi \cos v_{i}\right)-\int_{0}^{1}\left[\Gamma_{1}(\eta) F_{2}(\xi, 1)+\Gamma_{2}(\eta) F_{1}(\xi, 1)\right] \eta^{1 / 2} d \eta
\end{aligned}
$$

This constitutes a system of two Fredholm integral equations for the determination of the two functions $\Gamma_{i}(\xi)(j=1,2)$. The problem is essentially solved as the original 
unknown may be calculated from

$B(s)=\frac{(-1)^{i-1} \pi P_{j} a}{2\left(1-\kappa^{2}\right) s}\left[\Upsilon(1) J_{0}(s a)-\cos v_{i} \int_{0}^{1} \xi^{1 / 2} J_{0}(s a \xi) \Gamma(\xi) d \xi\right], \quad 0<\xi \leq 1$,

where

$$
\Upsilon(1)=-\delta \cos v_{i}=\cos v_{i} \int_{0}^{1} \xi^{1 / 2} \Gamma(\xi) d \xi .
$$

Case B-skew-symmetric problem. The scattering problem, in which tangential tractions

$$
\sigma_{y \nu}^{(r)}(x, 0)=-\sigma_{x y}^{(i)}(x, 0)=(-1)^{i} 2 \mu Q_{i} \exp \left(-i \alpha_{i} x \cos v_{i}\right), \quad|x|<a
$$

are applied to the crack surface, is said to be skew-symmetric with respect to the crack plane $y=0$. With $j=1,2$, the quantities $Q_{1}$ and $Q_{2}$ in Eq. (39) are related, respectively, to the amplitudes of the P-and SV-waves:

$$
Q_{1}=-\frac{1}{2} \alpha_{2}^{2} \phi_{0} \kappa^{2} \sin 2 v_{1}, \quad Q_{2}=\frac{1}{2} \alpha_{2}^{2} \psi_{0} \cos 2 v_{2} .
$$

Since the skew-symmetric problem may also be reduced to the solution of a system of coupled integrations in the same way as that of Case A, many of the detailed derivations will be omitted and only the essential steps will be given.

1. Tangential Tranctions Even in $x$. In this case, $\phi^{(r)}$ is odd in $x$ and $\psi^{(r)}$ even in $x$. Replacing $B_{i}(s)$ in Eqs. (28) by $C_{i}(s)(j=1,2)$, the vanishing of $\sigma_{y y}^{(r)}(x, 0)$ along the entire $x$-axis requires

$$
\left(s^{2}-\frac{1}{2} \alpha_{2}^{2}\right) C_{1}(s)-s \beta_{2} C_{2}(s)=0 .
$$

By having

$$
C(s)=s C_{1}(s)-\beta_{2} C_{2}(s)
$$

such that

$$
\left[\begin{array}{l}
C_{1}(s) \\
C_{2}(s)
\end{array}\right]=\frac{2 C(s)}{\alpha_{2}^{2} \beta_{2}}\left[\begin{array}{c}
s \beta_{2} \\
\left(s^{2}-\frac{1}{2} \alpha_{2}^{2}\right)
\end{array}\right]
$$

the boundary conditions in Eqs. (11) lead to

$$
\begin{aligned}
\frac{2}{\pi} \int_{0}^{\infty} C(s) \cos (s x) d s & =0, \quad|x|>a, \\
\frac{2}{\pi} \int_{0}^{\infty} m(s) C(s) \sin (s x) d s & =\frac{(-1)^{i} Q_{i} \sin \left(\alpha_{i} x \cos v_{i}\right)}{\alpha_{i} \cos v_{i}}, \quad|x|<a
\end{aligned}
$$

for the determination of $C(s)$. The function $m(s)$ is equal to $\left(\beta_{1} / \beta_{2}\right) f(s)$ with $f(s)$ being given previously by Eq. (15). The solution of Eqs. (40) is

$$
C(s)=\frac{\pi}{2} \int_{0}^{a} \tau \phi(\tau) J_{0}(s \tau) d \tau,
$$

where

$$
\begin{aligned}
\phi(\tau)=\left(1-\kappa^{2}\right)^{-1}\left[(-1)^{i-1} Q_{i} J_{0}\left(\alpha_{i} \tau \cos v_{i}\right)\right. & \\
& \left.+\int_{0}^{a} \zeta \phi(\zeta) d \zeta \int_{0}^{\infty} \operatorname{sn}(s) J_{0}(s \tau) J_{0}(s \zeta) d s\right], \quad 0<\tau \leq a
\end{aligned}
$$


is continuous on the interval $[0, a]$, and

$$
n(s)=m(s)+\left(1-\kappa^{2}\right) \rightarrow O\left(s^{-2}\right), \quad \text { as } s \rightarrow \infty .
$$

Performing the substitutions

$$
\xi=\frac{\tau}{a}, \quad \eta=\frac{\zeta}{a}, \quad \Phi(\xi)=\frac{(-1)^{i}\left(1-\kappa^{2}\right) \xi^{1 / 2} \phi(a \xi)}{Q_{i}}, \quad 0<\xi \leq 1
$$

on Eq. (42), there results a Fredholm integral equation

$$
\Phi(\xi)-\int_{0}^{1} \Phi(\eta) G(\xi, \eta) d \eta=(-1)^{i} \xi^{1 / 2} J_{0}\left(\alpha_{i} a \xi \cos v_{i}\right), \quad 0<\xi \leq 1
$$

whose kernel $G(\xi, \eta)$ is

$G(\xi, \eta)=\left(1-\kappa^{2}\right)^{-1}(\xi \eta)^{1 / 2} \int_{0}^{\infty} s n\left(\frac{s}{a}\right) J_{0}(s \xi) J_{0}(s \eta) d s, \quad 0<\xi \leq 1 ; \quad 0<\eta \leq 1$

For the purpose of computing the real and imaginary parts of $\Phi(\xi)$ numerically, a system of coupled integral equations can be deduced from Eq. (43):

$$
\begin{aligned}
& \Phi_{1}(\xi)-\int_{0}^{1}\left[\Phi_{1}(\eta) G_{1}(\xi, \eta)-\Phi_{2}(\eta) G_{2}(\xi, \eta)\right] d \eta=(-1)^{i} \xi^{1 / 2} J_{0}\left(\alpha_{j} a \xi \cos v_{i}\right), \\
& \Phi_{2}(\xi)-\int_{0}^{1}\left[\Phi_{1}(\eta) G_{2}(\xi, \eta)+\Phi_{2}(\eta) G_{1}(\xi, \eta)\right] d \eta=0, \quad 0<\xi \leq 1
\end{aligned}
$$

In Eq. (45), $G_{j}(\xi, \eta)(j=1,2)$ represent

$$
\begin{aligned}
& G_{1}(\xi, \eta)=(\xi \eta)^{1 / 2}\left[a^{2} N I_{0}(n a \xi) K_{0}(n a \eta)\right. \\
& \left.\quad+\int_{0}^{\infty} s q\left(\frac{s}{a}\right) J_{0}(s \xi) J_{0}(s \eta) d s\right], \quad 0<\xi \leq \eta \leq 1 \\
& G_{2}(\xi, \eta)=\frac{2}{\alpha_{2}^{2}\left(1-\kappa^{2}\right)}(\xi \eta)^{1 / 2} \int_{0}^{\infty} s\left[m_{2}\left(\frac{s}{a}\right)+n_{1}\left(\frac{s}{a}\right)\right] J_{0}(s \xi) J_{0}(s \eta) d s,
\end{aligned}
$$

where

$$
q(s)=1+2 \frac{f_{2}(s)-g_{1}(s)}{\alpha_{2}^{2}\left(1-\kappa^{2}\right)}-\frac{N}{s^{2}+n^{2}}
$$

and

$$
n^{2}=\frac{1}{2} \alpha_{2}^{2}\left(\frac{1+\kappa^{6}}{1+\kappa^{4}}\right), \quad N=\frac{1}{4} \alpha_{2}^{2}\left(\frac{1+\kappa^{4}}{1-\kappa^{2}}\right) .
$$

Finally, $C(s)$ is found:

$$
C(s)=\frac{(-1)^{i} \pi Q_{j} a}{2\left(1-\kappa^{2}\right) s}\left\{\Phi(1) J_{1}(s a)-\int_{0}^{1} \xi J_{1}(s a \xi)\left[\frac{\Phi(\xi)}{\xi^{1 / 2}}\right]^{\prime} d \xi\right\} .
$$

2. Tangential Traction Odd In $x$. When the tangential surface tractions on the crack are odd in $x$, Eqs. (13) apply provided that $A_{i}(s)$ are to be replaced by $D_{j}(s)(j=1,2)$. From the defined relationship

$$
D(s)=-s\left[s D_{1}(s)+\beta_{2} D_{2}(s)\right]
$$


and symmetry consideration, $D_{i}(s)$ are expressible in terms of $D(s)$ as

$$
\left[\begin{array}{l}
D_{1}(s) \\
D_{2}(s)
\end{array}\right]=\frac{2 D(s)}{\alpha_{2}^{2} \beta_{2} s}\left[\begin{array}{c}
-s \beta_{2} \\
\left(s^{2}-\frac{1}{2} \alpha_{2}^{2}\right)
\end{array}\right] .
$$

Now, the boundary conditions in Eqs. (11) require that $D(s)$ satisfies the dual integral equations

$$
\begin{aligned}
& \frac{2}{\pi} \int_{0}^{\infty} D(s) \cos (s x) d s=0, \quad|x|>a \\
& \frac{2}{\pi} \int_{0}^{\infty} m(s) D(s) \sin (s x) d s=i(-1)^{i-1} Q_{i} \sin \left(\alpha_{j} x \cos v_{j}\right), \quad|x|<a .
\end{aligned}
$$

Let $D(s)$ be connected with another function $\psi(\tau)$ through the relation

$$
D(s)=\frac{\pi}{2}\left[a \epsilon^{*} J_{0}(s a)+\int_{0}^{a} \psi(\tau) J_{0}(s \tau) d \tau\right]
$$

where

$$
\epsilon^{*}=-\frac{1}{a} \int_{0}^{a} \psi(\tau) d \tau
$$

Then, the second of equations (48) renders

$$
\begin{aligned}
& \psi(\tau)=\left(1-\kappa^{2}\right)^{-1} \tau\left\{i(-1)^{i} Q_{i} \alpha_{i} \cos v_{j} J_{0}\left(\alpha_{i} \tau \cos v_{i}\right)\right. \\
& \left.+\int_{0}^{\infty} \operatorname{sn}(s)\left[a \epsilon^{*} J_{0}(s a)+\int_{0}^{a} \psi(\zeta) J_{0}(s \zeta) d \zeta\right] J_{0}(s \tau) d s\right\}, \quad 0<\tau \leq a .
\end{aligned}
$$

Further, set $\xi=\tau / a, \eta=\zeta / a$ and adopt the notations

$$
\left[\begin{array}{c}
\epsilon \\
\xi^{1 / 2} \Psi(\xi)
\end{array}\right]=\frac{(-1)^{i}\left(1-\kappa^{2}\right)}{Q_{i} \cos v_{i}}\left[\begin{array}{c}
\epsilon^{*} \\
\Psi(a \xi)
\end{array}\right] .
$$

This carries Eq. (49) into

$\Psi(\xi)-\int_{0}^{1} \Psi(\eta) G(\xi, \eta) d \eta=i(-1)^{i-1} \alpha_{i} a \xi^{1 / 2} J_{0}\left(\alpha_{i} a \xi \cos v_{i}\right)+\epsilon G(\xi, 1), \quad 0<\xi \leq 1$

which can be separated into the following system of equations:

$$
\begin{aligned}
& \Psi_{1}(\xi)-\int_{0}^{1}\left[\Psi_{1}(\eta) G_{1}(\xi, \eta)-\Psi_{2}(\eta) G_{2}(\xi, \eta)\right] d \eta \\
& =-\int_{0}^{1}\left[\Psi_{1}(\eta) G_{1}(\xi, 1)-\Psi_{2}(\eta) G_{2}(\xi, \eta)\right] \eta^{1 / 2} d \eta, \quad 0<\xi \leq 1 \\
& \Psi_{2}(\xi)-\int_{0}^{1}\left[\Psi_{1}(\eta) G_{2}(\xi, \eta)+\Psi_{2}(\eta) G_{1}(\xi, \eta)\right] d \eta=(-1)^{i-1} \alpha_{j} a \xi^{1 / 2} J_{0}\left(\alpha_{i} a \xi \cos v_{j}\right) \\
& -\int_{0}^{1}\left[\Psi_{1}(\eta) G_{2}(\xi, 1)+\Psi_{2}(\eta) G_{1}(\xi, 1)\right] \eta^{1 / 2} d \eta
\end{aligned}
$$


The kernels $G_{i}(\xi, \eta)(j=1,2)$ are given by Eqs. (46). Once $\Psi(\xi)$ is known, $D(s)$ follows from

$$
D(s)=\frac{(-1)^{i-1} \pi Q_{i} a}{2\left(1-\kappa^{2}\right) s}\left[\Omega(1) J_{0}(s a)-\cos v_{i} \int_{0}^{1} \xi^{1 / 2} J_{0}(s a \xi) \Psi(\xi) d \xi\right],
$$

where

$$
\Omega(1)=-\epsilon \cos v_{i}=\cos v_{i} \int_{0}^{1} \xi^{1 / 2} \Psi(\xi) d \xi .
$$

Singular behavior of the near-field solution. The chief interest in the solution to the crack problem concerns its singular behavior at the crack points since it is the stresses and/or displacements in the vicinity of the crack that control the condition of crack propagation [17]. The singular character of these stresses for Cases A and B will be exhibited individually.

1. Case A. The symmetric crack-tip stresses of the scattered waves can be derived from Eqs. (13) and (28) which, when substituted into Eqs. (4), yield the expressions

$$
\begin{gathered}
\sigma_{x x}^{(r)}(x, y)=-\frac{4 \mu}{\pi} \int_{0}^{\infty}\left\{\left(\beta_{1}^{2}+\frac{1}{2} \alpha_{2}^{2}\right) \exp \left(-\beta_{1} y\right)\left[\begin{array}{l}
A_{1}(s) \\
B_{1}(s)
\end{array}\right]\right. \\
\left.+s \beta_{2} \exp \left(-\beta_{2} y\right)\left[\begin{array}{c}
A_{2}(s) \\
-B_{2}(s)
\end{array}\right]\right\}\left[\begin{array}{c}
\cos (s x) \\
\sin (s x)
\end{array}\right] d s \\
\begin{array}{r}
\sigma_{\nu \nu}^{(r)}(x, y)=\frac{4 \mu}{\pi} \int_{0}^{\infty}\left\{\left(s^{2}-\frac{1}{2} \alpha_{2}^{2}\right) \exp \left(-\beta_{1} y\right)\left[\begin{array}{c}
A_{1}(s) \\
B_{1}(s)
\end{array}\right]\right. \\
\left.+s \beta_{2} \exp \left(-\beta_{2} y\right)\left[\begin{array}{c}
A_{2}(s) \\
-B_{2}(s)
\end{array}\right]\right\}\left[\begin{array}{c}
\cos (s x) \\
\sin (s x)
\end{array}\right] d s \\
\sigma_{x \nu}^{(r)}(x, y)=\frac{4 \mu}{\pi} \int_{0}^{\infty}\left\{s \beta_{1} \exp \left(-\beta_{1} y\right)\left[\begin{array}{c}
A_{1}(s) \\
-B_{1}(s)
\end{array}\right]\right. \\
\left.+\left(s^{2}-\frac{1}{2} \alpha_{2}^{2}\right) \exp \left(-\beta_{2} y\right)\left[\begin{array}{c}
A_{2}(s) \\
B_{2}(s)
\end{array}\right]\right\}\left[\begin{array}{c}
\sin (s x) \\
\cos (s x)
\end{array}\right] d s .
\end{array}
\end{gathered}
$$

For an examination of the singular behavior of the stress components, it suffices to consider in $A_{i}(s)$ and $B_{i}(s)(j=1,2)$ the dominating terms

$$
\left[\begin{array}{l}
A_{1}(s) \\
A_{2}(s)
\end{array}\right]=\left[\begin{array}{c}
\frac{1}{2}-\left(s / \alpha_{2}\right)^{2} \\
s \beta_{1} / \alpha_{2}^{2}
\end{array}\right] \frac{(-1)^{i-1} \pi P_{i} a}{\beta_{1}\left(1-\kappa^{2}\right) s} \Lambda(1) J_{1}(s a)+\cdots
$$

and

$$
\left[\begin{array}{l}
B_{1}(s) \\
B_{2}(s)
\end{array}\right]=\left[\begin{array}{c}
-\frac{1}{2}+\left(s / \alpha_{2}\right)^{2} \\
s \beta_{1} / \alpha_{2}^{2}
\end{array}\right] \frac{(-1)^{j-1} \pi P_{j} a}{\beta_{1}\left(1-\kappa^{2}\right) s^{2}} \Upsilon(1) J_{0}(s a)+\cdots
$$

which contribute to the divergence of the improper integrals in Eqs. (53) at $x= \pm a$, $y=0$. This divergence is due to the behavior of the corresponding integrands as $s \rightarrow \infty$. Hence, asymptotic expansions of the integrands appearing in Eqs. (53) for large values of $s$ lead to 


$$
\begin{aligned}
& \sigma_{x x}^{(r)}(x, y)=\sigma_{j} a \int_{0}^{\infty}(s y-1) \exp (-s y)\left[\begin{array}{c}
\Lambda(1) \\
-\Upsilon(1)
\end{array}\right]\left[\begin{array}{c}
\cos (s x) J_{1}(s a) \\
\sin (s x) J_{0}(s a)
\end{array}\right] d s, \\
& \sigma_{y y}^{(r)}(x, y)=\sigma_{j} a \int_{0}^{\infty}(s y+1) \exp (-s y)\left[\begin{array}{c}
-\Lambda(1) \\
\Upsilon(1)
\end{array}\right]\left[\begin{array}{c}
\cos (s x) J_{1}(s a) \\
\sin (s x) J_{0}(s a)
\end{array}\right] d s, \\
& \sigma_{x y}^{(r)}(x, y)=-\sigma_{j} a \int_{0}^{\infty} s y \exp (-s y)\left[\begin{array}{c}
\Lambda(1) \\
\Upsilon(1)
\end{array}\right]\left[\begin{array}{c}
\sin (s x) J_{1}(s a) \\
\cos (s x) J_{0}(s a)
\end{array}\right] d s
\end{aligned}
$$

where $\sigma_{j}=(-1)^{i} 2 \mu P_{i}(j=1,2)$. The foregoing integrals can then be evaluated in closed elementary form by means of the well-known Bessel integral identities [16]

$$
\begin{aligned}
& \int_{0}^{\infty} \exp (-s y)\left[\begin{array}{c}
\cos (s x) \\
\sin (s x)
\end{array}\right] J_{0}(s a) d s=\frac{1}{\left(r_{1} r_{2}\right)^{1 / 2}}\left[\begin{array}{c}
\sin \frac{1}{2}\left(\theta_{1}+\theta_{2}\right) \\
\cos \frac{1}{2}\left(\theta_{1}+\theta_{2}\right)
\end{array}\right] \\
& \int_{0}^{\infty} s \exp (-s y)\left[\begin{array}{c}
\cos (s x) \\
\sin (s x)
\end{array}\right] J_{0}(s a) d s=-\frac{r}{\left(r_{1} r_{2}\right)^{3 / \overline{2}}}\left[\begin{array}{c}
\cos \left[\theta-\frac{3}{2}\left(\theta_{1}+\theta_{2}\right)\right] \\
\sin \left[\theta-\frac{3}{2}\left(\theta_{1}+\theta_{2}\right)\right]
\end{array}\right]
\end{aligned}
$$

etc. The polar coordinates $(r, \theta),\left(r_{1}, \theta_{1}\right)$, and $\left(r_{2}, \theta_{2}\right)$ have the meaning shown in Figure 1. In the limit as $r_{2} \rightarrow 2 a, r \rightarrow a$, and $\theta_{2}=\theta \rightarrow 0$, the unbounded contributions to the total stress field near $x=+a, y=0$ are precisely equal to those of the scattered waves since the stress field of the incident waves are nonsingular. Carrying out the limiting process just mentioned yields

$$
\begin{aligned}
& \sigma_{x x}=\frac{k_{1}}{\left(2 r_{1}\right)^{1 / \overline{2}}} \cos \left(\frac{1}{2} \theta_{1}\right)\left[1-\sin \left(\frac{1}{2} \theta_{1}\right) \sin \left(\frac{3}{2} \theta_{1}\right)\right]+O(1) \\
& \sigma_{y y}=\frac{k_{1}}{\left(2 r_{1}\right)^{1 / \overline{2}}} \cos \left(\frac{1}{2} \theta_{1}\right)\left[1+\sin \left(\frac{1}{2} \theta_{1}\right) \sin \left(\frac{3}{2} \theta_{1}\right)\right]+O(1) \\
& \sigma_{x y}=\frac{k_{1}}{\left(2 r_{1}\right)^{1 / \overline{2}}} \cos \left(\frac{1}{2} \theta_{1}\right) \sin \left(\frac{1}{2} \theta_{1}\right) \cos \left(\frac{3}{2} \theta_{1}\right)+O(1),
\end{aligned}
$$

where

$$
k_{1}=\left[\begin{array}{l}
\Lambda(1) \\
\Upsilon(1)
\end{array}\right] \sigma_{j} a^{1 / 2}
$$

It is apparent that the same angular distribution in $\theta_{1}$ and the characteristic inverse square-root singularity, $r_{1}^{-1 / 2}$, at the crack tip are observed in both the statical and dynamical stress solutions. In the dynamic case, however, the strength of this stress singularity, measured by $k_{1}$, fluctuates in time and is proportional to the circular frequency, amplitude of input wave, angle of incidence, etc. Adopting the criterion in the theory of brittle fracture [17], $k_{1}$ may be regarded as a dynamic stress-intensity factor the critical value of which is a function of material property and can be measured experimentally to determine the point of incipient fracture.

The influence of fluctuating loads upon the $k_{1}$-factor can be brought out more clearly by a plot of $\left|k_{1} / \sigma_{1} a^{1 / 2}\right|$ versus normalized wave number $\alpha_{1} a$ at normal incidence, $v_{1}=$ $\pi / 2$, such that the crack is excited by P-waves only. In this case, $\Upsilon$ in Eq. (56) is zero. Referring to Fig. 2, the static solution is given by $k_{1}=\sigma_{1} a^{1 / 2}$ which corresponds to $\omega=0$. Here, $\sigma_{1}$ represents the magnitude of the static tensile stress applied in the direction normal to the crack plane. As $\omega$ increases from zero, the requisite numerical results are obtained by solving the integral equation for $\Lambda$ on an electronic computer for values of 


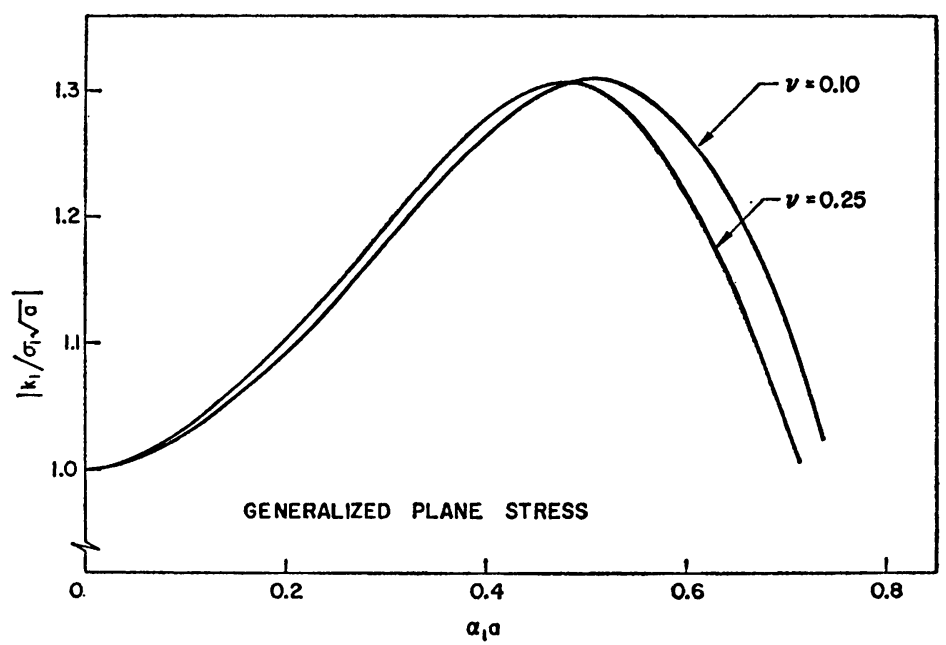

Fig. 2. P-waves at normal incidence $\left(\nu_{1}=90^{\circ}\right)$.

Poisson's ratio $\nu=0.10$ and $\nu=0.25$ under the conditions of generalized plane stress. Note that for $\nu=0.10$ the maximum value of $\left|k_{1} / \sigma_{1} a^{1 / 2}\right|$ is 1.31 at $\alpha_{1} a \simeq 0.51$ and beyond this point the curve declines quickly. The peak of the $\left|k_{1} / \sigma_{1} a^{1 / 2}\right|$-curve for $\nu=0.25$ is slightly lower and occurs at $\alpha_{1} a \simeq 0.48$.

2. Case B. By a similar procedure, the local stress distribution for the skew-symmetric case may be obtained by taking

$$
\left[\begin{array}{l}
C_{1}(s) \\
C_{2}(s)
\end{array}\right]=\left[\begin{array}{c}
-s \beta_{2} / \alpha_{2}^{2} \\
\frac{1}{2}-\left(s / \alpha_{2}\right)^{2}
\end{array}\right] \frac{(-1)^{i-1} \pi Q_{j} a}{\beta_{2}\left(1-\kappa^{2}\right) s} \Phi(1) J_{1}(s a)+\cdots
$$

and

$$
\left[\begin{array}{l}
D_{1}(s) \\
D_{2}(s)
\end{array}\right]=\left[\begin{array}{c}
-s \beta_{2} / \alpha_{2}^{2} \\
-\frac{1}{2}+\left(s / \alpha_{2}\right)^{2}
\end{array}\right] \frac{(-1)^{j-1} \pi Q_{j} a}{\beta_{2}\left(1-\kappa^{2}\right) s^{2}} \Omega(1) J_{0}(s a)+\cdots \cdot
$$

Without repeating the details, the singular stresses are found to be

$$
\begin{aligned}
& \sigma_{x x}=-\frac{k_{2}}{\left(2 r_{1}\right)^{1 / 2}} \sin \left(\frac{1}{2} \theta_{1}\right)\left[2+\cos \left(\frac{1}{2} \theta_{1}\right) \cos \left(\frac{3}{2} \theta_{1}\right)\right]+O(1), \\
& \sigma_{\nu y}=\frac{k_{2}}{\left(2 r_{1}\right)^{1 / 2}} \sin \left(\frac{1}{2} \theta_{1}\right) \cos \left(\frac{1}{2} \theta_{1}\right) \cos \left(\frac{3}{2} \theta_{1}\right)+O(1), \\
& \sigma_{x y}=\frac{k_{2}}{\left(2 r_{1}\right)^{1 / 2}} \cos \left(\frac{1}{2} \theta_{1}\right)\left[1-\sin \left(\frac{1}{2} \theta_{1}\right) \sin \left(\frac{3}{2} \theta_{1}\right)\right]+O(1) .
\end{aligned}
$$

The parameter $k_{2}$ is

$$
k_{2}=\left[\begin{array}{l}
\Phi(1) \\
\Omega(1)
\end{array}\right] \tau_{i} a^{1 / 2},
$$

where $\tau_{i}=(-1)^{i} 2 \mu Q_{i}$, and it has the same physical meaning as $k_{1}$. At normal incidence, $v_{i}=\pi / 2$, and $\Omega=0$, the static solution of $k_{2}=\tau_{2} a^{1 / 2}$ is recovered as $\omega \rightarrow 0$, and $\tau_{2}$ may be interpreted as the uniform shear stress at infinity.

Let the crack be excited by SV-waves and consider a state of plane strain. As is evident 
from Fig. 3, the variation of $\left|k_{2} / \tau_{2} a^{1 / 2}\right|$ with $\alpha_{2} a$, the normalized wave number for shear waves, follows the same trend as that of the $\left|k_{1} / \sigma_{1} a^{1 / 2}\right|$-curves in Fig. 2 . It is seen that the peaks of the curves in Fig. 3 become progressively lower as the Poisson's ratio is increased

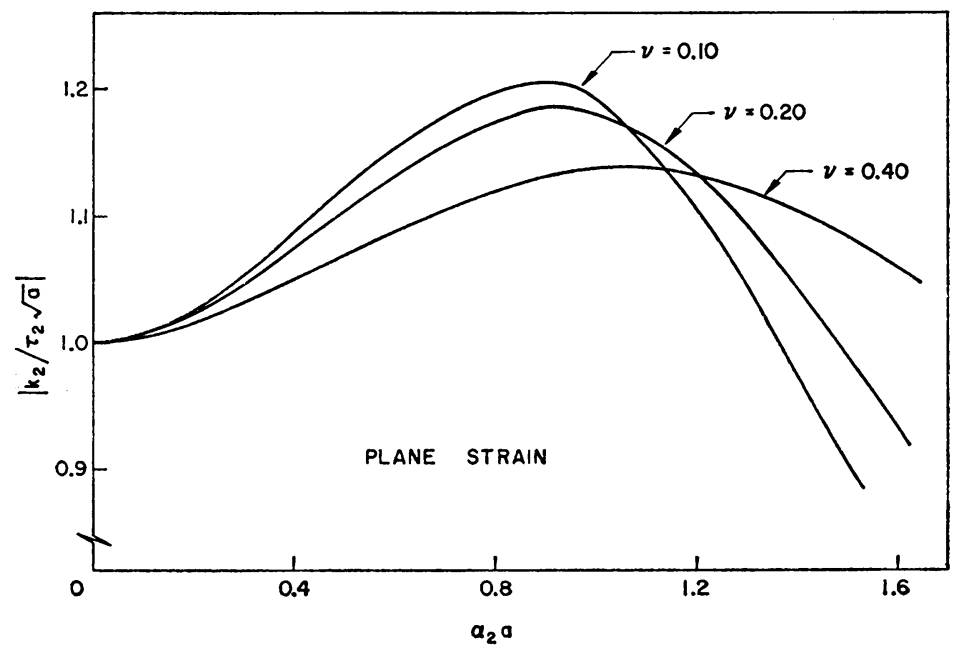

FIG. 3. SV-waves at normal incidence $\left(\nu_{2}=90^{\circ}\right)$.

from $\nu=0.10$ to $\nu=0.40$. At $\nu=0.10$, the peak occurs at $\alpha_{2} a \simeq 0.9$ and is approximately $20.5 \%$ greater than the static value.

Further insight into the effects of different types of input waves on the intensity of the dynamic stresses may be gained from Fig. 4, which depicts the absolute value of the

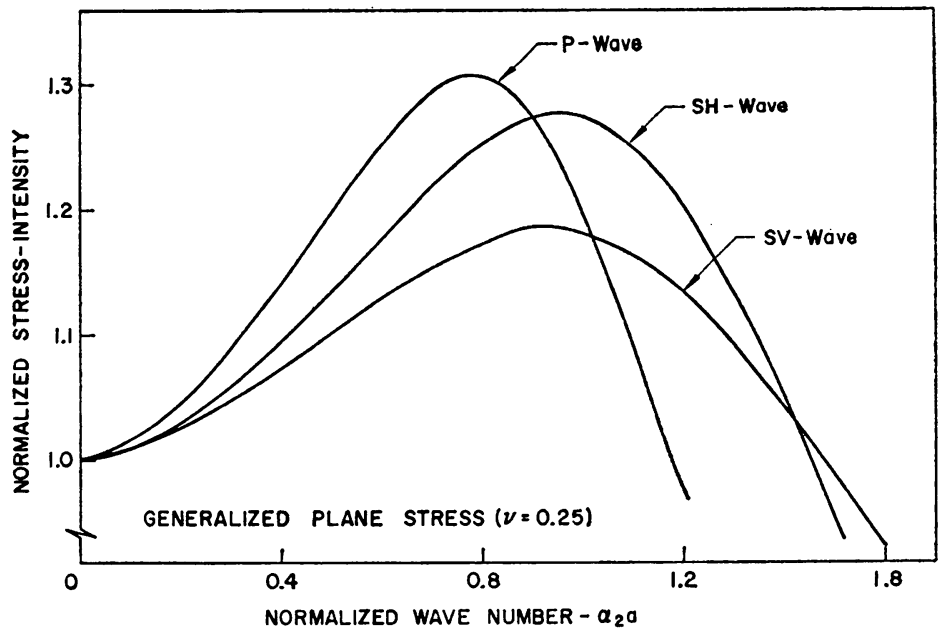

FIG. 4. Near-field stress solutions for three types of input waves.

dimensionless stress-intensity factor as a function of $\alpha_{2} a$ for $\nu=0.25$. The greatest amplification of the stress-intensity factor corresponds to the P-waves, and the lowest to the SV-waves. Between these two lies the curve for the SH-waves producing anti-plane 
type of deformation at the crack tip. The data for this curve were taken from the work of Loeber and Sih [9] for the purpose of comparison.

Discussions. As remarked earlier, if a medium containing a crack is subjected to fluctuating loads, the crack-surface displacement may alternate in sign depending upon whether the incident field is tensile or compressive. Consequently, the relative displacement of the upper and lower surfaces of the crack can be negative in the compressive case which causes the opposing crack surfaces to come into contact. When this happens, the tractions and displacements are continuous across the line crack invalidating the original assumption of a traction-free crack. To avoid this situation, an additional tensile field must be added onto the crack to keep the crack surfaces separated. The requisite tensile field can be estimated from the displacement solution of the original problem of scattered waves.

For tractions that are applied symmetrically to the crack plane, the quantity of interest is the normal displacement

$$
\begin{aligned}
\frac{(-1)^{i} a\left(1-\kappa^{2}\right) u_{y}(x, 0)}{P_{i}}= & {\left[1-(x / a)^{2}\right]^{1 / 2} \Lambda(1)-\int_{x / a}^{1}\left[\xi^{2}-(x / a)^{2}\right]\left[\frac{\Lambda(\xi)}{\xi^{1 / 2}}\right]^{\prime} d \xi } \\
& +\cos v_{i}\left[\frac{\pi}{2} \int_{0}^{x / a} \xi^{1 / 2} \Gamma(\xi) d \xi+\int_{x / a}^{1} \sin ^{-1}\left(\frac{x}{a \xi}\right) \xi^{1 / 2} \Gamma(\xi) d \xi\right. \\
& \left.-\sin ^{-1}\left(\frac{x}{a}\right) \int_{0}^{1} \xi^{1 / 2} \Gamma(\xi) d \xi\right], \quad|x|<a .
\end{aligned}
$$

The numerical calculation of Eq. (59) will be carried out for the case of normal incidence, $v_{1}=\pi / 2$. Fig. 5 displays the deformed shapes of the crack for $\alpha_{1} a=0.0,0.3,0.5$, and 0.7 . In order to satisfy the zero traction condition, the crack opening shown by the curves in Fig. 5 should at least be doubled so that sufficient clearance is provided to prevent the crack surfaces from closing on the compression cycle. As expected, the maximum value of $\left|a\left(1-\kappa^{2}\right) u_{y}(x, 0) / P_{1}\right| \simeq 1.25$ occurs at $\alpha_{1} a \simeq 0.5$, the normalized wave number at which the dimensionless crack-tip stress-intensity factor $\left|k_{1} / \sigma_{1} a^{1 / 2}\right|$ is also a maximum.

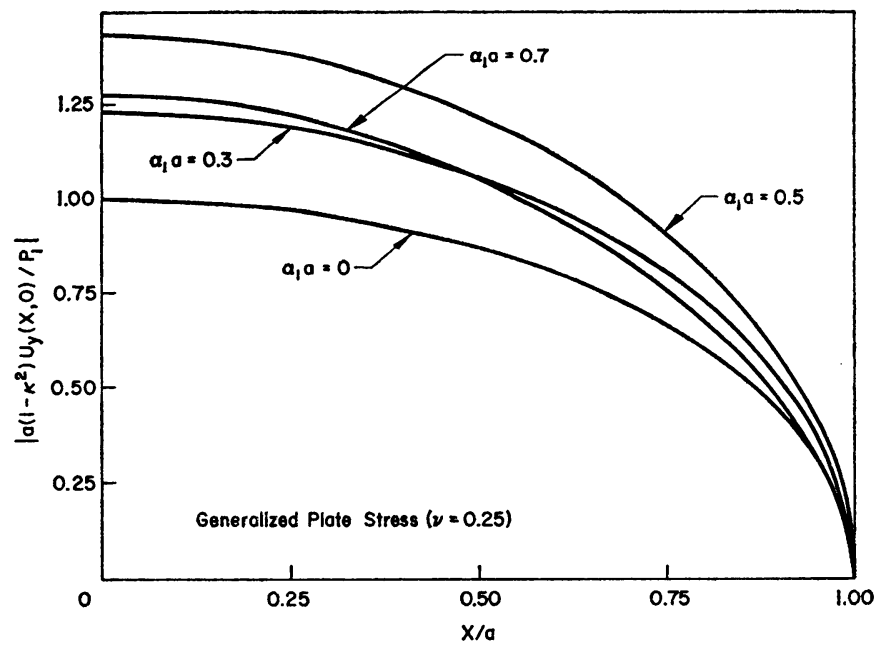

FIg. 5. Transverse displacement of crack surface. 
To recapitulate, it should be mentioned that except for certain angles of incidence, the general input wave of the type in Eq. (5) or Eq. (7) will produce scattered waves composing both $\mathrm{P}$ - and SV-waves. A complete solution of the diffraction problem, therefore, necessitates both the symmetric (Case A) and skew-symmetric (Case B) treatments outlined in the paper. Only in the particular situations of normally incident waves does it suffice to consider only one part of the solution, namely Case A or Case B. To be noted further is that by superimposing a number of input waves, it is possible to generate other initial states of stresses which reduce, for low wave numbers, to familiar static problems. For instance, the sum of two compressional waves at normal and grazing incidence given by

$$
\phi^{(i)}=\phi_{0}\left[\exp \left(-i \alpha_{1} y\right)+\exp \left(-i \alpha_{1} x\right)\right] \exp (-i \omega t), \quad \psi^{(i)}=0
$$

and

$$
\phi^{(i)}=\phi_{0}\left[\exp \left(-i \alpha_{1} y\right)-\nu \exp \left(-i \alpha_{1} x\right)\right] \exp (-i \omega t), \quad \psi^{(i)}=0
$$

produces dynamic stress-states which reduce to the biaxial stress state

$$
\sigma_{x x}=\sigma_{y y}=(1+\nu) \sigma_{1}
$$

and the uniaxial stress state

$$
\sigma_{x x}=0, \quad \sigma_{y y}=\left(1-\nu^{2}\right) \sigma_{1}
$$

respectively in the static limit.

Although the diffraction problem heretofore considered was that of a single incident wave which gave rise to sinusoidal type of boundary conditions on the line of disconcontinuity, i.e.,

$$
\left[\begin{array}{l}
\sigma_{y y}^{(r)}(x, 0) \\
\sigma_{x y}^{(r)}(x, 0)
\end{array}\right] \sim\left[\begin{array}{c}
\cos \left(\alpha_{j} x \cos v_{j}\right) \\
\sin \left(\alpha_{j} x \cos v_{j}\right)
\end{array}, \quad|x|<a,\right.
$$

the method of solution may be generalized to cover response to an arbitrary periodic disturbance by expanding the boundary function $(s)$ in Fourier series as follows:

$$
\sigma_{\nu \nu}^{(r)}(x, 0)=\sum_{n=0}^{\infty} \epsilon_{n}\left[A_{n}^{*} \cos \left(\frac{n \pi x}{a}\right)+B_{n}^{*} \sin \left(\frac{n \pi x}{a}\right)\right], \quad|x|<a .
$$

The symbol $\epsilon_{n}$ takes the value unity for $n=0$ and two for $n \geq 1$. The coefficients in the Fourier series are obtainable from the familiar integrals

$$
\begin{array}{ll}
A_{n}^{*}=\frac{1}{2 a} \int_{-a}^{a} \sigma_{\nu y}^{(r)}(x, 0) \cos \left(\frac{n \pi x}{a}\right) d x, & n=0,1,2, \cdots \\
B_{n}^{*}=\frac{1}{2 a} \int_{-a}^{a} \sigma_{\nu \nu}^{(r)}(x, 0) \sin \left(\frac{n \pi x}{a}\right) d x, & n=1,2, \cdots
\end{array}
$$

and $B_{0}^{*}=0$. Similar expressions for the tangential traction $\sigma_{x y}^{r}(x, 0)$ can be written down.

It is evident that the present method can also be employed to solve a number of other problems dealing with the reflection of electromagnetic or acoustic waves by a finite line of discontinuity. 


\section{ReFerences}

[1] P. M. Morse and H. Feshbach, Methods of theoretical physics. Part II, McGraw-Hill, New York, 1955

[2] D. S. Jones, The theory of electromagnetism, Macmillan, New York, 1964

[3] V. A. Fock, Electromagnetic diffraction and propagation problems, Pergamon Press, New York, 1965

[4] Y. H. Pao, Dynamic stress concentration in an elastic plate, Appl. Mech. 29, 299-305 (1962)

[5] Y. H. Pao and C. C. Mow, Dynamic stress concentration in an elastic plate with rigid circular inclusion, Proceedings of Fourth U. S. National Congress of Applied Mechanics 1, 335-345 (1962)

[6] S. A. Thau and Y. H. Pao, Stress-intensification near a semi-infinite rigid-smooth strip due to diffraction of elastic waves, J. Appl. Mech. 34, 119-126 (1967)

[7] B. Noble, Methods based on the Wiener-Hopf technique, Pergamon Press, New York, 1958

[8] G. C. Sih, Some elastodynamic problems of cracks, Internat. J. Fracture Mech. 4, 51-68 (1968)

[9] J. F. Loeber and G. C. Sih, Diffraction of anti-plane shear waves by a finite crack, Acoust. Soc. Amer. 44, 90-98 (1968)

[10] D. Ang and L. Knopoff, Diffraction of scalar tlastic waves by a finite crack, Proc. Nat. Acad. Sci. 51, 593-598 (1964)

[11] R. A. Schmeltzer and M. Lewin, Function-theoretic solution to a class of dual integral equations and an application to diffraction theory, Quart. Appl. Math. 21, 269-283 (1964)

[12] J. W. Miles, Homogeneous solutions in elastic wave propagation, Quart. Appl. Math. 18, 37 (1960)

[13] M. Papadopoulos, Diffraction of plane elastic waves by a crack with application to a problem of brittle fracture, J. Austral. Math. Soc. 3, Part 3, 325-339 (1963)

[14] N. I. Muskhelishvili, Some basic problems of mathematical theory of elasticity, P. Noordhoff, Holland, 1953

[15] E. T. Whittaker and G. N. Watson, Modern analysis, 4th Ed., Cambridge University Press, London, 1962

[16] G. N. Watson, Theory of Bessel functions, 2nd Ed., Cambridge University Press, London, 1958

[17] G. C. Sih and H. Liebowitz, Mathematical theories of brittle fracture, in Mathematical fundamentals of fracture, Vol. 2, 67-190, Academic Press, New York, 1968 